\title{
Ventilatory thresholds during wheelchair exercise in individuals with spinal cord injuries
}

\author{
KD Coutts and DC McKenzie \\ School of Human Kinetics and Allan McGavin Sports Medicine Centre, University of British Columbia, \\ 6081 University Blvd, Vancouver BC, Canada V6T 1Z1
}

\begin{abstract}
The ventilatory thresholds of 30 male wheelchair athletes were determined from their respiratory and metabolic responses to a continuously progressive exercise protocol to peak oxygen uptake on a wheelchair ergometer. The peak oxygen uptake (peak VO2), ventilatory threshold in $1 \mathrm{~min}^{-1}$ (VTL), and ventilatory threshold expressed as a percentage of peak VO2 (VT\%) were measured for all subjects. Statistical analyses of selected subsamples were used to note sport and functional ability level differences in these variables. Analysis of peak $\mathrm{VO} 2$ by functional classification (old International Stoke Mandeville Games Federation system; classes 1A-5, plus an amputee class) indicated that paraplegic (classes 2-5) and the amputee class athletes $\left(2.48 \mathrm{lmin}^{-1}\right)$ were higher than tetraplegic (classes $1 \mathrm{~A}-1 \mathrm{C})$ athletes $\left(0.95 \mathrm{~min}^{-1}\right)$. The paraplegic and amputee classes were combined for a comparison of peak $\mathrm{VO} 2$ by sport which showed that track athletes $\left(2.80 \mathrm{lmin}^{-1}\right)$ were higher than basketball players $\left(2.411 \mathrm{~min}^{-1}\right)$ who were higher than athletes from other sports $\left(1.881 \mathrm{~min}^{-1}\right)$. The VTL analyses demonstrated differences similar to the peak VO2 analyses. The VT\% analyses, however, showed no sport differences, but the tetraplegic athletes had higher VT\% values $(87 \%)$ than the paraplegic plus amputee group $(69 \%)$.
\end{abstract}

Keywords: tetraplegia; paraplegia; ventilatory thresholds; wheelchairs; exertion; sports

\section{Introduction}

Relatively recent reviews indicate the growing body of information on the peak exercise responses of wheelchair dependent individuals in general and wheelchair athletes in particular. ${ }^{1,2}$ Little information, however, is available on their responses to submaximal exercise and/or relationships between exercise intensity and response variables. The anaerobic and/or ventilatory threshold has become a key parameter in exercise studies since it has been related to a significant increase in anaerobic metabolism as exercise intensity increases. In analyzing respiratory responses to progressive exercise, Coutts et $^{3} l^{3}$ noted that the absolute value of the anaerobic threshold occurred at progressively higher oxygen uptakes in tetraplegic persons, high lesion paraplegic individuals, and low lesion paraplegic persons, respectively. Their analysis was limited to group mean data, however, and Davis ${ }^{4}$ has noted the error in not using individual data for threshold determination. More recently, Lakomy et al ${ }^{5}$ reported a high relationship $(r=0.83)$ between the wheelchair speed eliciting a blood lactate concentration of $4 \mathrm{mmoll}^{-1}$ and $5 \mathrm{~km}$ time trial performance in a group of wheelchair athletes. Their study supports the importance of the level of exercise producing an elevation of the anaerobic energy production as a determinant of endurance performance in wheelchair dependent individuals. A third study by Flandrois et al ${ }^{6}$ using arm crank ergometry rather than wheelchair propulsion found that the lactate threshold, expressed as a percentage of maximal oxygen uptake, was higher in a trained paraplegic group compared to able bodied controls, and higher in high lesion paraplegics compared to low lesion paraplegics. They attributed the paraplegic versus control difference to local muscle adaptations of the paraplegics in response to training.

The present study was undertaken to extend this previous work on the anaerobic threshold of wheelchair athletes. Ventilatory threshold differences related to sport participation, and level of injury including tetraplegic, paraplegic, and amputee persons were analyzed.

\section{Methods}

Thirty-one male subjects who normally used a manually propelled wheelchair for daily activities and/or competitive sports participation were recruited according to a protocol approved by the institution's ethical review committee, and each subject provided written informed consent. The distribution of the subjects with regard to sport and functional ability are presented in Table 1. The older International Stoke Mandeville Games Federation classification system ${ }^{1}$ was used to group the subjects by functional ability since it provided an initial 
Table 1 Subject count by sport and classification

\begin{tabular}{lcccc}
\hline Class/Sport & Track & Basketball & Other & Total \\
\hline 1A-Tetraplegic & 2 & 0 & 0 & 2 \\
1B-Tetraplegic & 2 & 0 & 2 & 4 \\
1C-Tetraplegic & 0 & 0 & 3 & 3 \\
2-Paraplegic & 2 & 0 & 2 & 4 \\
3-Paraplegic & 1 & 3 & 0 & 4 \\
4-Paraplegic & 4 & 1 & 2 & 7 \\
5-Paraplegic & 1 & 2 & 0 & 3 \\
AMP & 3 & 0 & 1 & 4 \\
Total & 15 & 6 & 10 & 31 \\
\hline
\end{tabular}

separation of tetraplegic individuals into three categories (classes 1A, 1B and 1C) and paraplegic persons into four (classes 2-5). Amputee persons (AMP), all with double, above knee amputations, were also initially separated for analysis purposes. The sport designation in Table 1 indicates the number of subjects who competed at the national or international level in track (and/or road racing) or basketball, with the 'other' designation used for subjects who did not fit into these groups, but were generally active in other sports including swimming, table tennis, and target shooting.

Each subject used their own sport specific wheelchair in completing a continuous, progressive exercise test to peak oxygen uptake on a set of rollers following a warm-up. Subjects in the tetraplegic classes began propelling their wheelchairs at 20 pushes per minute in synchronization with a calibrated metronome and push frequency was increased by 10 pushes per minute each minute until peak oxygen uptake was attained. For all other subjects, wheeling started at 40 pushes per minute with the frequency increased by 20 each minute up to 80 pushes per minute. This was followed by resistance increases of $0.37 \mathrm{~N} \cdot \mathrm{m}$ each minute on the rear roller by means of a friction strap connected to a calibrated sinus balance until the subject achieved a peak oxygen uptake. Oxygen uptake was determined every $15 \mathrm{~s}$ throughout the test using a calibrated, open circuit system, and peak oxygen uptake values were taken as the highest average over four consecutive $15 \mathrm{~s}$ intervals. Respiratory exchange ratios greater than 1.05 (all subjects), and heart rates above 165 beats per minute (only paraplegic and AMP subjects) were used to ascertain that a valid peak oxygen uptake had been achieved.

Ventilatory thresholds were determined for each subject from visual inspection of plots of the $15 \mathrm{~s}$ interval values for minute ventilation (VE), ventilatory equivalent for oxygen ( $\mathrm{VE} / \mathrm{VO} 2)$, ventilatory equivalent for carbon dioxide (VE/VCO2), and carbon dioxide elimination (VCO2) versus oxygen uptake (VO2) by two observers. The primary criteria used in identifying the ventilatory threshold was an increase in $\mathrm{VE} / \mathrm{VO} 2$ while $\mathrm{VE} / \mathrm{VCO} 2$ remained relatively constant or decreased as described by Beaver et al.$^{7}$ The point of steeper increases in $\mathrm{VCO} 2$ vs $\mathrm{VO} 2$ and then $\mathrm{VE}$ vs VO2 were used, if needed, to aid in determining the threshold. The two observers initially viewed the plots independently and complete agreement in determining ventilatory threshold was noted for 24 of the 31 subjects, and an overall interobserver correlation of 0.95 was found. One subject, a class $1 \mathrm{~B}$, other sport athlete, did not demonstrate a clear ventilatory threshold and his data were not included in the data summaries nor data analyses. The two observers either agreed on a single value for the VTL or an average of the independently determined values was used for the remaining six subjects.

Data analyses were carried out to compare sport and functional group differences in the peak oxygen uptake, VTL, and VT\%. The lack of subjects in some of the cells in Table 1, however, did not permit a single, complete analysis of variance across both of these factors. Thus, a one way analysis of variance for the effect of ability classification was run which combined subjects across sports, while sport differences were tested using just the paraplegic and amputee class athletes. The 0.05 level of significance was accepted as indicative of an effect in each analysis of variance, and Newman-Keul's statistic was used to make further between group contrasts when appropriate.

\section{Results}

Table 2 presents the three dependent variables, peak oxygen uptake, the absolute value of the ventilatory threshold (VTL) in $1 \mathrm{~min}^{-1}$, and the VTL expressed as a percentage of the peak VO2 (VT\%), for the subjects analyzed by functional classification. A graphic presentation of the VT\% variable by functional classification is contained in Figure 1. All variables demonstrated a significant class effect, and follow-up contrasts indicated that all tetraplegic classes $(1 \mathrm{~A}, 1 \mathrm{~B}$, and $1 \mathrm{C})$ were lower than all of the paraplegic classes (2-5 and AMP) for peak VO2. VTL was lower in the tetraplegic classes only in comparison to classes 3,4 , and 5 , while VT\% was higher in the tetraplegic classes when contrasted with classes 2, 4, 5, and AMP. The analysis of these variables and inspection of plots such as Figure 1 suggested a relatively clear separation of these subjects into tetraplegic and paraplegic groups. A secondary

Table 2 Data by classification

\begin{tabular}{lccc}
\hline Class & \multicolumn{3}{c}{ Mean $($ SE $)$} \\
\cline { 2 - 4 } & $\begin{array}{c}\text { PeakVO2 } \\
\left(\text { lmin }^{-1}\right)\end{array}$ & $\begin{array}{c}\text { VTL } \\
\left(\text { lmin }^{-1}\right)\end{array}$ & $\begin{array}{c}\text { VT\% } \\
(\% \text { of peak })\end{array}$ \\
\hline 1A & $1.09(0.17)$ & $0.93(0.28)$ & $84(6)$ \\
1B & $0.83(0.12)$ & $0.69(0.22)$ & $85(5)$ \\
1C & $1.03(0.14)$ & $0.95(0.24)$ & $92(5)$ \\
2 & $1.95(0.25)$ & $1.36(0.20)$ & $69(4)$ \\
3 & $2.43(0.29)$ & $1.78(0.20)$ & $74(4)$ \\
4 & $2.73(0.22)$ & $1.89(0.15)$ & $69(3)$ \\
5 & $2.63(0.34)$ & $1.72(0.24)$ & $66(5)$ \\
AMP & $2.53(0.29)$ & $1.64(0.20)$ & $66(4)$ \\
F-value & 9.43 & 4.44 & 4.68 \\
p(F) & $<0.0001$ & 0.003 & 0.002 \\
\hline
\end{tabular}




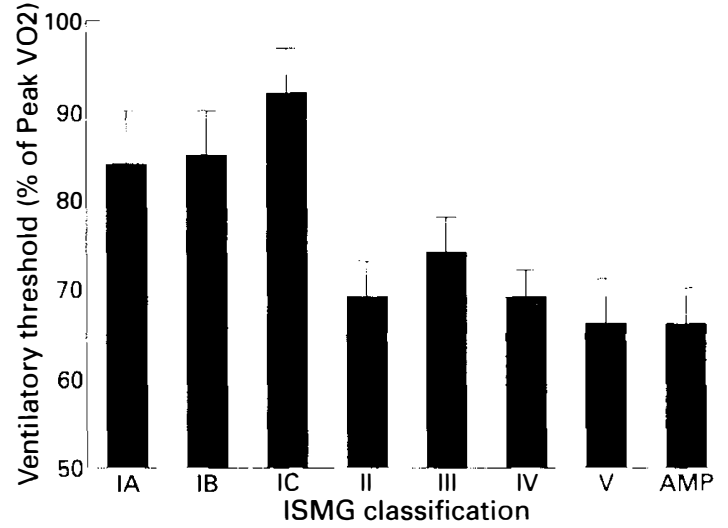

Figure 1 Means and standard errors of ventilatory threshold as a percentage of peak VO2 by functional classification

analysis of this data with subjects regrouped into just tetraplegic and paraplegic groups indicated significantly lower peak VO2 (0.95 vs $\left.2.481 \mathrm{~min}^{-1}\right)$ and VTL (0.85 vs $\left.1.71 \mathrm{lmin}^{-1}\right)$, and higher $\mathrm{VT} \%$ (87 vs $\left.69 \%\right)$, respectively.

Differences between paraplegic subjects by sport participation are noted in Table 3. No significant difference was found in VT\%, while peak VO2 and VTL exhibited a sport effect. Post hoc contrasts indicated that the track athletes were significantly $(P<0.05)$ higher than the 'other' athletes, while the bastketball palyers tended $(P<0.10)$ to be different from both these groups in peak VO2 and VTL.

\section{Discussion}

The peak VO2 results in this sample of wheelchair athletes are generally consistent with previous studies. ${ }^{1,2}$ That is, higher peak VO2 values are observed in subjects with a greater amount of functional muscle mass following an injury or illness. A direct relationship between the aerobic demands of the wheelchair sport and its associated level of training with peak $\mathrm{VO} 2$ has also been noted. This agreement with previous data provides an indication that the present sample is a reasonably representative sample of wheelchair athletes, and therefore the findings of this study should be generalizable to other wheelchair athletes.

The subject differences in the absolute value of the ventilatory threshold (VTL in $1 \mathrm{~min}^{-1}$ ) by sport and

Table 3 Paraplegic and amputee data by sport

\begin{tabular}{lccc}
\hline Sport & \multicolumn{3}{c}{ Mean (SE) } \\
\cline { 2 - 4 } & $\begin{array}{c}\text { Peak VO2 } \\
\left(\text { lmin }^{-1}\right)\end{array}$ & $\begin{array}{c}\text { VTL } \\
\left(\text { lmin }^{-1}\right)\end{array}$ & $\begin{array}{c}\text { VT\% } \\
\text { (\% of peak) }\end{array}$ \\
\hline Track & $2.80(0.14)$ & $1.95(0.11)$ & $70(3)$ \\
Basketball & $2.41(0.19)$ & $1.62(0.15)$ & $68(4)$ \\
Other & $1.88(0.21)$ & $1.26(0.16)$ & $68(4)$ \\
F-value & 6.60 & 6.37 & 0.10 \\
$\mathrm{p}(\mathrm{F})$ & 0.007 & 0.008 & 0.91 \\
\hline
\end{tabular}

functional classification, as expected, closely followed the peak VO2 analyses. A generally strong positive correlation has been shown between VTL and peak $\mathrm{VO} 2$ in previous studies, ${ }^{4}$ and a correlation of 0.93 between these variables was found in the current relatively heterogeneous wheelchair athlete sample. Therefore, little additional information about subject differences can be obtained from the analysis of VTL that was not noted in the peak $\mathrm{VO} 2$ analysis.

The relationship between peak $\mathrm{VO} 2$ and the relative ventilatory threshold (VT\%) is less clear and therefore VT\% represents a variable that is more distinct from the peak VO2. A correlation of -0.54 between peak VO2 and VT\% was noted in the current total sample with an $r=-0.12$ when using a more homogeneous subsample of the subjects in just the paraplegic classes. The VT\% values were higher than those reported for able bodied subjects during arm crank ergometry, ${ }^{8}$ but this difference could be attributed to exercise mode rather than sample differences. Flandrois et $a l^{6}$ also using arm crank exercise, have noted a higher lactate threshold, expressed as a percentage of maximal oxygen uptake, in a paraplegic sample versus an able bodied control group (59 vs 43\%), and high lesion paraplegics had a higher value than low lesion paraplegics (63vs 54\%). The average of $69 \%$ for the VT\% in the current male paraplegic subsample is somewhat higher than the $59 \%$ of Flandrois et al, ${ }^{6}$ but this difference may be attributed to the use of gas exchange versus blood lactate measurements to determine the threshold value and/or the difference in mode of exercise used.

In a group of 10 paraplegic and two tetraplegic wheelchair athletes wheeling a $5 \mathrm{~km}$ time trial on a treadmill, Lakomy et $a l^{5}$ observed that the tetraplegics were able to wheel at about $90 \%$ of their VO2 max while the paraplegics averaged $76 \%$ during this endurance performance. These values are in reasonable agreement with the average $\mathrm{VT} \%$ for tetraplegics of $87 \%$ and paraplegics of $69 \%$ in the present study. Lakomy $e^{2} a^{5}$ also noted that the average speed during the $5 \mathrm{~km}$ trial was not significantly different from the speed eliciting a blood lactate concentration of $4 \mathrm{mmoll}^{-1}$ in their athletes. The finding of a significantly higher ventilatory threshold, as a percentage of peak VO2, in tetraplegics compared to paraplegics in the present study is thus consistent with the limited evidence from previous studies.

While determination of the mechanism for a higher, relative ventilatory threshold in tetraplegics is beyond the scope of the present study, a few possible models can be considered on the basis of the current results. The general relationship of ventilatory threshold to lactate threshold and anaerobic metabolism has been previously noted and discussed. ${ }^{4,9}$ The higher relative threshold of tetraplegic persons, compared to paraplegic persons, could be ascribed to their lower functional muscle mass in relation to their total blood volume. That is, it would be expected that tetraplegic persons would have a lower peak lactate production capacity due to their lower functional muscle mass which would 
lead to lower peak blood lactate values. Eriksson et al $^{10}$ have reported lower blood lactic acid levels in tetraplegic persons compared to paraplegic persons and able bodied controls following maximal exercise involving wheelchair propulsion. Thus, tetraplegic persons with a maximal exercise blood lactate of 5-7 $\mathrm{mmol}^{-1}$ would have to be at a higher percentage of maximal effort in order to stimulate respiratory compensation for metabolic acidosis than paraplegic persons. This line of reasoning, however, would also suggest that amputees persons with a higher functional muscle mass to total blood volume ratio compared to paraplegic persons should exhibit a significantly higher blood lactate and the assumed ventilatory consequences at a lower intensity of exercise. The lack of a significant different in $\mathrm{VT} \%$ between the AMP and any of the paraplegic classes does not support this possible link between blood lactate and the ventilatory threshold in this sample.

The clear distinction between the tetraplegic and paraplegic groups in VT\% with no apparent trends within these groups as shown in Figure 1 suggests that the lower $\mathrm{VT} \%$ in paraplegic persons involves some difference created by spinal lesions at or above $\mathrm{C} 8$ (classes 1A, 1B, and 1C) $v s$ those at or below $\mathrm{T} 1$ (classes 2-5). Two possibilities are the loss of central sympathetic control to the heart ${ }^{11}$ and the loss of central innervation of the intercostal muscles that are richly supplied with afferent muscle spindles. ${ }^{12}$ Hypotheses to explain the noted delay or modified increase in the ventilatory drive of tetraplegic persons to a higher percentage of maximal exercise could be made that involve either or both of these losses. An alternate viewpoint is that the individuals with tetraplegia have a low peak VO2 in relation to their ventilatory threshold rather than a high ventilatory threshold in relation to their peak VO2. The lack of a clear ventilatory threshold in one of the class $1 \mathrm{~B}$ (lesion $\mathrm{C} 7$ ) non-endurance sport athletes with a peak VO2 of $0.891 \mathrm{~min}^{-1}$ indicates that the peak VO2 may be below the ventilatory threshold in some individuals with higher spinal cord lesions. Further study of the respiratory and metabolic responses to progressive exercise of individuals with a spinal cord injury, thus, seems promising in identifying some of their intact physiological control mechanisms and the contribution of specific mechanisms to the ventilatory threshold.

\section{References}

1 Shephard RJ. Sports medicine and the wheelchair athlete. Sports Med 1988; 4: 226-247.

2 Wells CL, Hooker SP. The spinal injured athlete. Adapt Phys Activ $Q$ 1990; 7: 265-285.

3 Coutts KD, Rhodes EC, McKenzie DC. Submaximal exercise responses of tetraplegics and paraplegics. J Appl Physiol 1985; 59: 237-241.

4 Davis JA. Anaerobic threshold: review of the concept and directions for future research. Med Sci Sports Exerc 1985; 17: 6-18.

5 Lakomy HKA, Campbell I, Williams C. Treadmill performance and selected physiological characteristics of wheelchair athletes. Br J Sports Med 1987; 21: 130-133.

6 Flandrois $\mathrm{R}$ et al. Aerobic performance capacity in paraplegic subjects. Eur J Appl Physiol 1986; 55: 604-609.

7 Beaver WL, Wasserman K, Whipp BJ. A new method for detecting anaerobic threshold by gas exchange. J Appl Physiol 1986; 60: 2020-2027.

8 Davis JA et al. Anaerobic threshold and maximal aerobic power for three modes of exercise. J Appl Physiol 1976; 41: 544-550.

9 Brooks GA. Anaerobic threshold: review of the concept and directions for future research. Med Sci Sports Exerc 1985; 17: 22-31.

10 Eriksson P, Lofstrom L, Ekblom B. Aerobic power during maximal exercise in untrained and well-trained persons with quadriplegia and paraplegia. Scand J Rehabil Med 1988; 20: 141-147.

11 Frankel HL, Mathias CJ. The cardiovascular system in tetraplegia and paraplegia. In: Vinken PJ, Bruyn GW (eds). Handbook of Clinical Neurology. North Holland: Amsterdam, 1976, pp 313-333.

12 Fugl-Meyer AR. The respiratory system. In: Vinken PJ, Bruyn GW (eds). Handbook of Clinical Neurology. North Holland: Amsterdam, 1976, pp 335-353. 